\title{
Interplay between morphology and magnetoelectric coupling in Fe/PMN-PT multiferroic heterostructures studied by microscopy techniques
}

\author{
Federico Motti $\odot,{ }^{1,2, *, \dagger}$ Giovanni Vinai $\odot,{ }^{1}$ Valentina Bonanni $\odot,{ }^{1,2}$ Vincent Polewczyk $\odot,{ }^{1}$ Paola Mantegazza, ${ }^{2}$ \\ Thomas Forrest, ${ }^{3}$ Francesco Maccherozzi, ${ }^{3}$ Stefania Benedetti $\odot,{ }^{4}$ Christian Rinaldi $\odot, 5,6$ Matteo Cantoni $\odot,{ }^{5}$ \\ Damiano Cassese, ${ }^{7}$ Stefano Prato, ${ }^{7}$ Sarnjeet S. Dhesi, ${ }^{3}$ Giorgio Rossi, ${ }^{1,2}$ Giancarlo Panaccione $\odot,{ }^{1}$ and Piero Torelli ${ }^{1}$ \\ ${ }^{1}$ Istituto Officina dei Materiali (IOM-CNR), Laboratorio TASC, Area Science Park, S.S. 14 km 163.5, 34149 Trieste, Italy \\ ${ }^{2}$ Department of Physics, Università degli Studi di Milano, Via Celoria 16, 20133 Milano, Italy \\ ${ }^{3}$ Diamond Light Source, Harwell Science and Innovation Campus, Didcot OX11 ODE, United Kingdom \\ ${ }^{4}$ CNR, Istituto Nanoscienze, via G. Campi 213/a, 41125 Modena, Italy \\ ${ }^{5}$ Dipartimento di Fisica, Politecnico di Milano, Via G. Colombo 81, 20131 Milano, Italy \\ ${ }^{6}$ Istituto di Fotonica e Nanotecnologie (IFN-CNR), piazza Leonardo da Vinci 32, 20133 Milano, Italy \\ ${ }^{7}$ A.P.E. Research srl, Area Science Park, S.S. 14 km 163.5, 34149 Trieste, Italy
}

(Received 21 July 2020; revised 4 October 2020; accepted 29 October 2020; published 25 November 2020)

\begin{abstract}
A ferromagnetic (FM) thin film deposited on a substrate of $\mathrm{Pb}\left(\mathrm{Mg}_{1 / 3} \mathrm{Nb}_{2 / 3}\right) \mathrm{O}_{3}-\mathrm{PbTiO}_{3}(\mathrm{PMN}-\mathrm{PT})$ is an appealing heterostructure for the electrical control of magnetism, which would enable nonvolatile memories with ultralow-power consumption. Reversible and electrically controlled morphological changes at the surface of PMN-PT suggest that the magnetoelectric effects are more complex than the commonly used "strain-mediated" description. Here we show that changes in substrate morphology intervene in magnetoelectric coupling as a key parameter interplaying with strain. Magnetic-sensitive microscopy techniques are used to study magnetoelectric coupling in Fe/PMN-PT at different length scales, and compare different substrate cuts. The observed rotation of the magnetic anisotropy is connected to the changes in morphology, and mapped in the crack pattern at the mesoscopic scale. Ferroelectric polarization switching induces a magnetic field-free rotation of the magnetic domains at micrometer scale, with a wide distribution of rotation angles. Our results show that the relationship between the rotation of the magnetic easy axis and the rotation of the in-plane component of the electric polarization is not straightforward, as well as the relationship between ferroelectric domains and crack pattern. The understanding and control of this phenomenon is crucial to develop functional devices based on FM/PMN-PT heterostructures.
\end{abstract}

DOI: $10.1103 /$ PhysRevMaterials.4.114418

\section{INTRODUCTION}

The electric-field control of magnetism is desirable for many applications, especially low-power and nonvolatile memories [1-5]. Single-phase multiferroics (displaying more than one long-range ferroic order, like ferromagnetic and ferroelectric [6]) would be suitable materials for these purposes, but generally the ordering temperatures are too low to be of practical interest $[7,8]$. To circumvent this, artificial heterostructures are studied, in which piezo/ferroelectrics are coupled with magnetic materials through an interface [9-15]. These systems can provide room-temperature functionality and are suitable for industrial production. Several review articles have appeared on the topic over the last decade [16-20]. As thoroughly discussed in those papers, the main concerns are the understanding and description of the phenomena at the origin of magnetoelectric coupling in heterostructures, in order to improve and tailor their functionality.

\footnotetext{
${ }^{*}$ Corresponding author: Paul Scherrer Institute, Villigen, Switzerland

†federico.motti@psi.ch
}

One way to induce magnetoelectric coupling is through interface strain; an electric field induces a deformation of the heterostructure by the inverse piezoelectric effect; the strain transferred across the interface to the magnetic layer determines a change of magnetic anisotropy (and eventually magnetic domain rotation) via inverse magnetostriction. In this respect, the relaxor ferroelectric $\mathrm{Pb}\left(\mathrm{Mg}_{1 / 3} \mathrm{Nb}_{2 / 3}\right) \mathrm{O}_{3}-\mathrm{PbTiO}_{3}$ (PMN-PT) is very appealing, because of its high piezoelectric coefficient.

The solid solution $\mathrm{PMN}_{1-x}-\mathrm{PT}_{x}$ shows a complex phase diagram as a function of $x$ [21] [see Fig. 1(a)]. At the morphotropic phase boundary $(0.3<x<0.4)$, the compound exhibits the highest piezoelectric coefficient and a coexistence of rhombohedral and tetragonal phases [22,23]. $\mathrm{PMN}_{0.7}-\mathrm{PT}_{0.3}$ is a substrate widely used in multiferroic heterostructures [24-27]. It is rhombohedral, with the electric dipole moment oriented along the eight possible $\langle 111\rangle$ directions. In the (011) cut, application of an electric field out of plane can effectively stabilize different in-plane strain states at remanence [28]. This can be used to modify the magnetic state of a film (or nanostructures) deposited on top of it [29-31]. Considering shear strain, it has been shown that a rotation of magnetization $\mathbf{M}$ by an angle of about $62^{\circ}$ is expected when the 

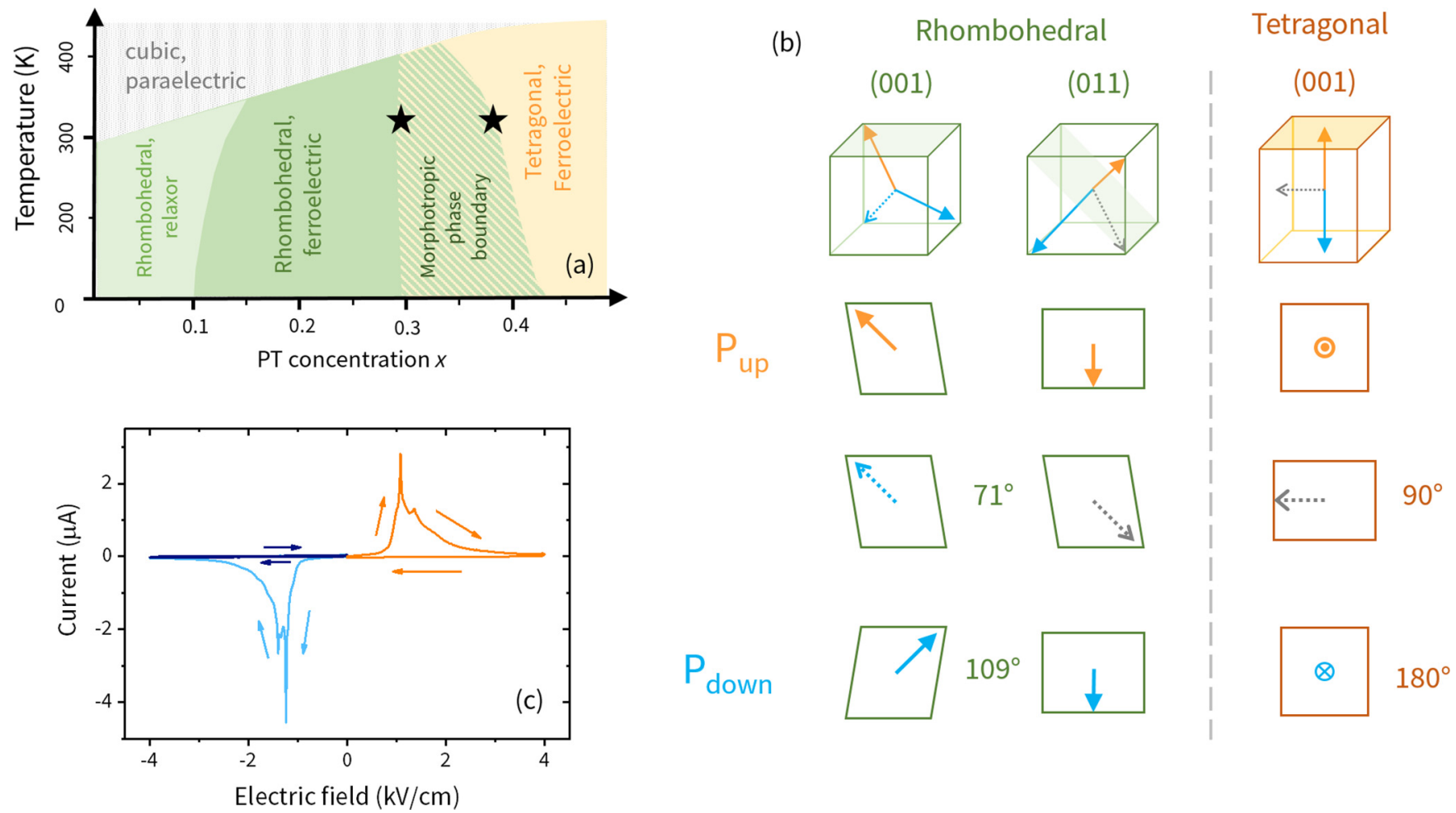

FIG. 1. (a) Schematic of the phase diagram of $\mathrm{PMN}_{1-x}-\mathrm{PT}_{x}$. Stars mark the investigated compositions. (b) Sketch of the possible directions of the ferroelectric polarization $P$ in the pseudocubic unit cell. The surface plane of the substrate is highlighted in the 3D representation. The 2D representations show the projection of $P$ on this plane. Orange and blue colors represent up/down out-of-plane direction of $P$, respectively. (c) Two-terminal current vs electric field characteristic measured during the out-of-plane poling of PMN-PT substrates. The presence of peaks in the current testifies the switch of $P$ at the coercivity of the substrate. After setting the polarization, subsequent sweep of the voltage with the same polarity does not produce any peak (dark line for negative electric field values), which demonstrates that the polarization state has a net remanence.

polarization $\mathbf{P}$ switches from out of plane to in plane with a rotation of $71^{\circ}$ or $109^{\circ}$ [32]. In the (001) cut instead, the in-plane strain at remanence is modified only if the $\mathbf{P}$ rotates by $109^{\circ}$, while no changes take place for rotations of $71^{\circ}$ [see Fig. 1(b)]. This leads to an observed copresence of volatile and nonvolatile magnetoelectric effects [33-35]. In general however the change in anisotropy is mainly visible at angles oriented $45^{\circ}$ with respect to the pseudocubic axes [36], due to the orientation of $\mathbf{P}$ (and hence of the transferred strain) along the $\langle 111\rangle$ directions. The magnetic easy axis is expected to rotate by $90^{\circ}$, purely due to shear-strain effects.

In 2018, Liu et al. reported a surprising mechanical behavior of $\mathrm{PMN}_{0.72}-\mathrm{PT}_{0.28}(001)$ crystals close to the morphotropic phase boundary: out-of-plane electric polarization produced cracks on the surface, which can be erased and reformed reversibly and reproducibly by toggling the polarity of the applied field, with possible applications as resistive memory [37]. Shortly after, our group showed how this reversible change in morphology has strong implications on the magnetoelectric effect in ferromagnet/PMN $\mathrm{PN}_{0.6}-\mathrm{PT}_{0.4}(001)$ heterostructures [38]. Both reports show that the surface cracking of the substrate breaks the interfacing metallic film when switching the polarization out of plane. However, when the polarity is reversed, the cracks disappear. Consequently, the metallic film recovers its conductive and magnetic properties.

In this paper, we report results on the morphological changes connected with magnetoelectric coupling in
$\mathrm{Fe} / \mathrm{PMN}_{1-x}-\mathrm{PT}_{x}$. Our results reveal a correspondence between rotation of the magnetic anisotropy and crack orientation. We employ microscopy techniques to unveil the microscopic details of the observed effect in $\mathrm{Fe} / \mathrm{PMN}_{0.6}-\mathrm{PT}_{0.4}(001)$. Magneto optical Kerr effect (MOKE) microscopy allows us to measure the local variations in the shape of the ferromagnetic hysteresis loop. In particular, we compare different regions separated by cracks. Complementary, x-ray magnetic circular dichroism combined with photoemission electron microscopy (XMCD-PEEM) allows us to reconstruct the spatially resolved rotation of the magnetization vector $\mathbf{M}$, in connection with the switching of the electrical polarization $\mathbf{P}$. The comparison with a different substrate cut and composition, $\mathrm{PMN}_{0.7}-\mathrm{PT}_{0.3}(011)$, points out that the interplay between morphology and magnetoelectric coupling is a general phenomenon in ferromagnet/PMN-PT heterostructures.

\section{RESULTS}

Out-of-plane electrical polarization of the $\mathrm{PMN}_{0.6}-\mathrm{PT}_{0.4}(001)$ substrates induces morphology changes that emerge at the surface as vertically displaced areas separated by cracks. These cracks reversibly annihilate for an applied electric field of inverted polarity. Cracked and smooth morphologies correspond hence to opposite orientations of $\mathbf{P}$ (from here on, $P_{\text {up }}$ and $P_{\text {down }}$, respectively). The change is evident at the coercive electric field $\left[E_{\mathrm{C}} \approx 1.5 \mathrm{kV} / \mathrm{cm}\right.$, see 

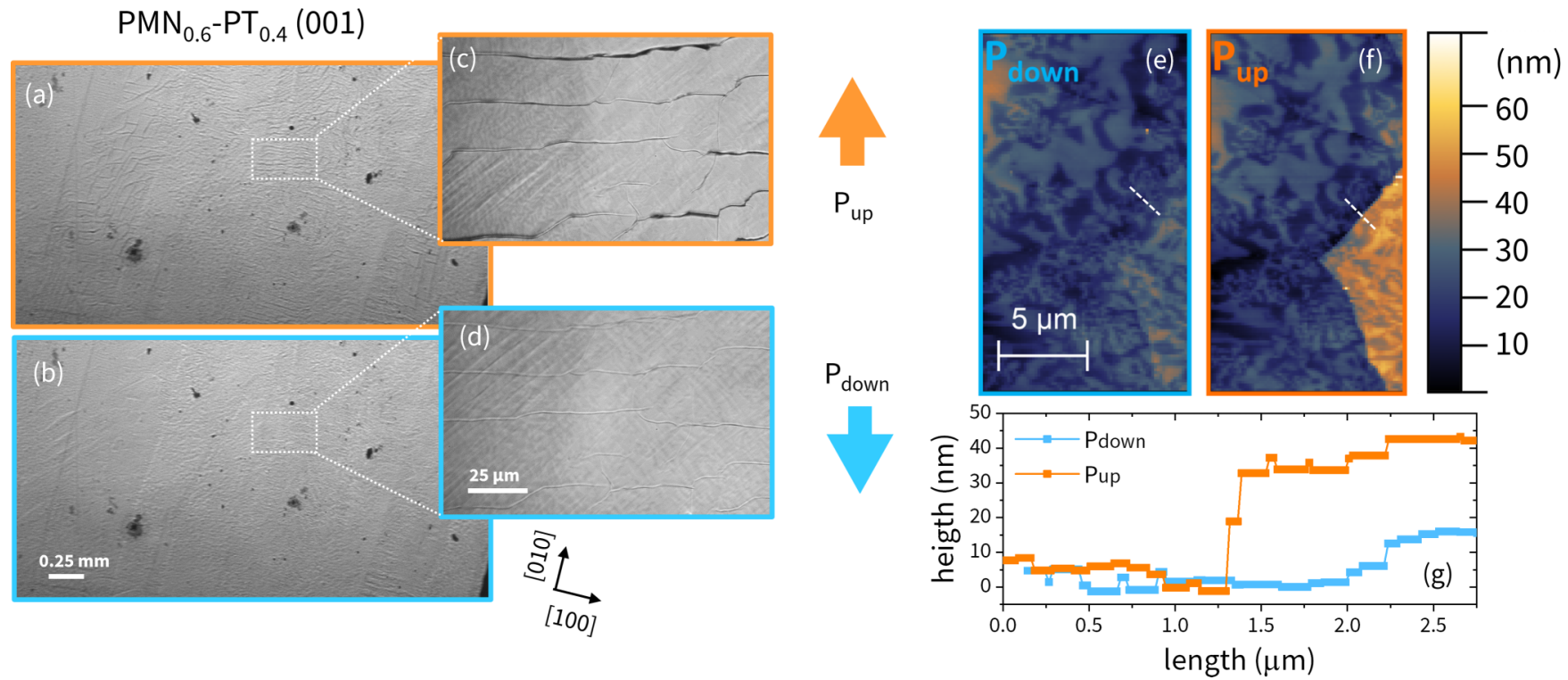

FIG. 2. Electrically driven change in morphology of PMN-PT(001). (a) Optical microscope image in the cracked state, and (b) after reversing the polarization. (c), (d) Higher-magnification images of a region of the surface, in which the cracks form an ordered pattern. (e), (f) AFM topographic images acquired at zero bias, after switching the electric polarization down and up, respectively. (g) Cross section of the previous images across the step [dashed white line in (e) and (f)].

Fig. 1(c)]). The change in morphology can be observed with an optical microscope. Figure 2 shows some typical images for PMN-PT (001), in $P_{\text {up }}$ and $P_{\text {down }}$ states (orange and light blue squares, respectively). Cracks form a dense and irregular network (panel a), and they all seem to be reversible (panel b). Within a millimeter distance, the motif formed by the cracks may vary considerably. Occasionally, we can observe a stripelike pattern (panel $\mathrm{c}$, showing a magnified image of a region in panel a). In the $P_{\text {down }}$ state, faint lines are still visible in correspondence of the cracks (panel d, in the same area as panel b).

The cracking induces the formation of steps and terraces on the surface. Figure 2 (panels e, $\mathrm{f}$, and $\mathrm{g}$ ) shows a quantitative characterization by Atomic Force Microscopy (AFM). On PMN-PT (001), the step height is of the order of tens of nanometers, although terraces up to $100 \mathrm{~nm}$ high were observed [38].

\section{A. Kerr microscopy characterizations}

In $\mathrm{PMN}_{0.6}-\mathrm{PT}_{0.4}(001)$ the cracking pattern has a typical length scale of $10-50 \mu \mathrm{m}$, which is suitable for a study by Kerr microscopy. In particular, we analyzed the change in the magnetic hysteresis loop in regions separated by a crack. We selected two different regions where cracks run parallel [see Figs. 3(a) and 3(b)]. These stripelike patterns in the two regions are roughly perpendicular one to the other. We stress once again that these two regions are quite peculiar, since on most of the surface the cracks form a disordered pattern in PMN-PT (001) [see Fig. 1(c)]; nonetheless, it can be considered as a simplified case.

A first analysis was performed by integrating the magnetic signal on a large region of interest, containing three cracks [dashed boxes in Figs. 3(a) and 3(b)]. The results can be seen in Fig. 3 (panels c-f). We notice that the effect of the electric
$\mathrm{Fe} / \mathrm{PMN}_{0.6}-\mathrm{PT}_{0.4}(001)$

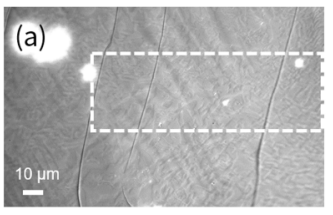

vertical cracks

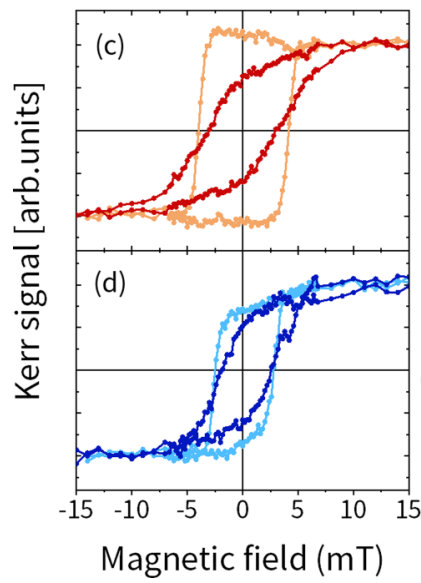

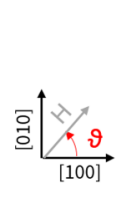

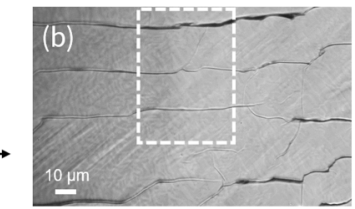

horizontal cracks

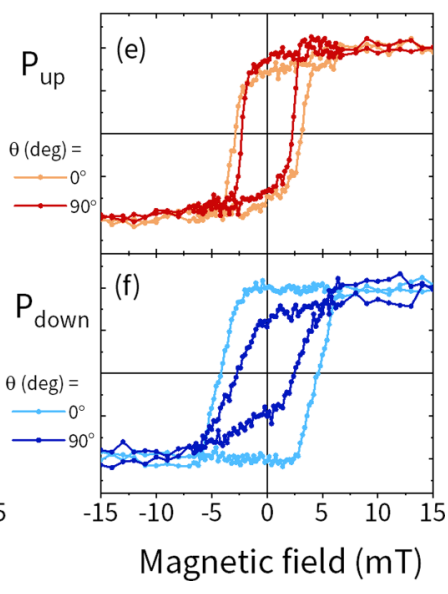

FIG. 3. (a) Microscope image of a region with cracks running parallel, labeled as "vertical". The dashed box delimits the integration area used to obtain the hysteresis loops. (b) Image of a second region in which the cracks are almost perpendicular to the first one, labeled as "horizontal". (c) Hysteresis loops acquired for polarization up in the region shown in panel a. (d) Same for polarization down. (e), (f) Analogous measurements in the region shown in panel b. Different directions of the magnetic field $\left(\vartheta=0^{\circ}\right.$ or $\left.90^{\circ}\right)$ correspond to light or dark colors, respectively. 


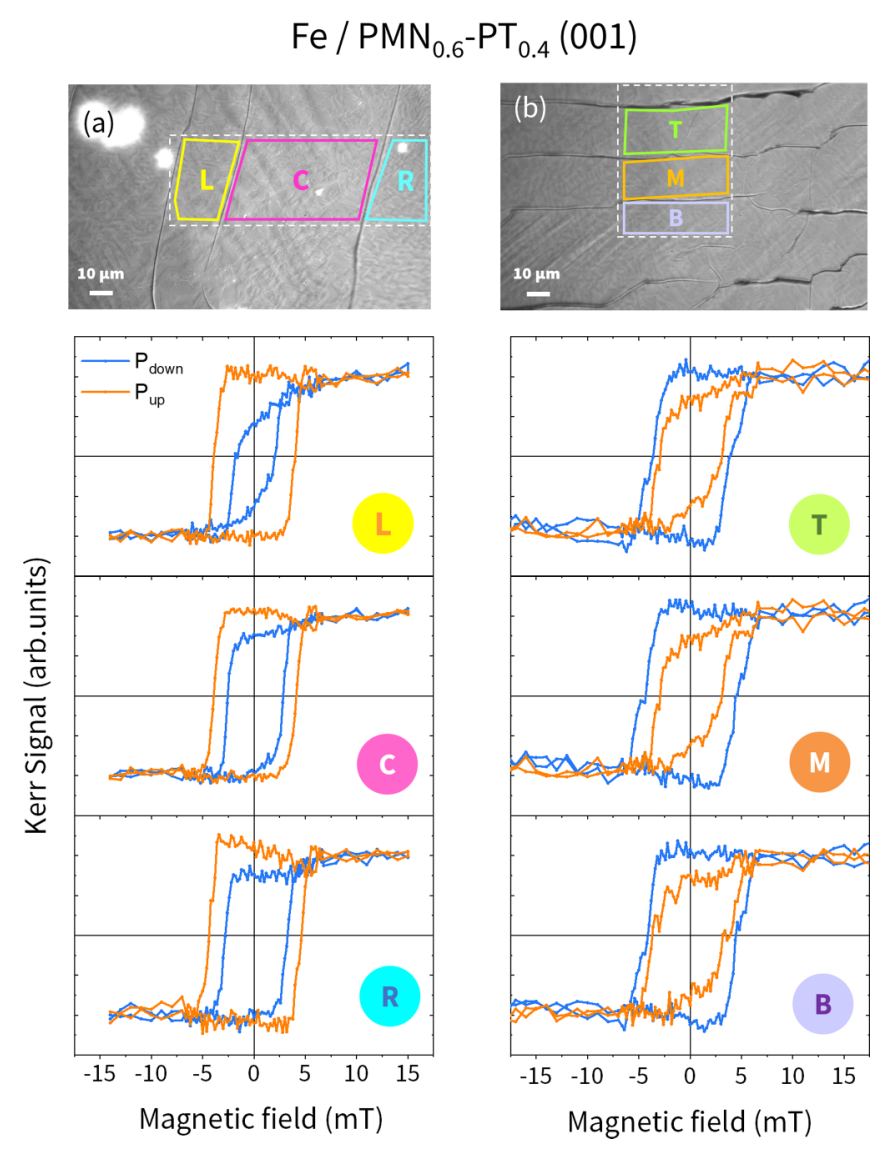

FIG. 4. Stripe-by-stripe analysis of the magnetic behavior for the two regions with (a) vertical cracks and (b) horizontal cracks. The large regions of interest (white dashed contours) were divided in subregions (colored contours, labeled Left-Center-Right and TopMiddle-Bottom) and the intensity was integrated over each of them to obtain the hysteresis loops. For all measurements the magnetic field was applied along $\vartheta=0^{\circ}$.

polarization is opposite in the two investigated regions. For the region with vertical cracks, the $P_{\text {up }}$ state is more anisotropic than $P_{\text {down }}$ (compare panels c and d), whereas for the region with horizontal cracks it is the opposite, and $P_{\text {down }}$ is more anisotropic than $P_{\text {up }}$ (compare panels e and f).

In a second analysis, we integrated separately the intensity coming from each subregion separated by cracks. The aim is to understand if the cracking of the surface induces different magnetic anisotropy between the two sides of the crack. The results are presented in Fig. 4 for the case of $\vartheta=0^{\circ}$. It can be seen that the different "stripes" within the same region display hysteresis loops that are not identical, with variations in the shape of the hysteresis [see in Fig. 4(a) the changes in the $P_{\text {down }}$ state from left to right]. Nonetheless, the overall effect of the electric polarization on the anisotropy is uniform. Thus, the magnetoelectric coupling acts in similar ways in regions separated by cracks, coherently contributing to the overall behavior shown in Fig. 3 .

\section{B. Imaging of ferromagnetic domains by PEEM}

After probing magnetoelectric coupling at the macroscopic and mesoscopic scale, we employed XMCD-PEEM to reach submicrometer resolution $[11,32,39,40]$ and to study the evolution of magnetic domains as a function of out-of-plane ferroelectric polarization of the substrate. With a field of view of $50 \mu \mathrm{m}$, the imaged area is comparable with the one probed by XMCD measurements [42]. We can therefore use PEEM to have a "spatially resolved insight" of the previously reported data [38]. Two-dimensional (2D) maps of the magnetization were obtained at zero electric bias, before and after switching the polarization between $P_{\text {up }}$ and $P_{\text {down }}$ in situ on a demagnetized sample. Here we present two zones of the same sample whose starting demagnetized $P_{\text {down }}$ states were strongly different, investigating the role of surface crack in the $P_{\text {up }}$ state.

In the first case, shown in Fig. 5, the starting magnetic configuration was almost saturated. Figure 5(a) shows the polarization-averaged intensity map acquired at the $\mathrm{Fe} L_{3}$ preedge in $P_{\text {up }}$ state, where a surface crack is visible (highlighted by the red dashed box). Its position is marked by a dashed white line in the magnetization vector maps [39] in panels $b, c$, and $d$, where the direction of $\mathbf{M}$ is mapped via a color code. We arbitrarily choose $\vartheta=0^{\circ}$ as the most represented direction of $\mathbf{M}$ in the initial state to determine the rotation angles. The initial polarization state was set to $P_{\text {down }}$ ex situ, before demagnetizing the sample. In this initial state [Fig. 4(b)] the magnetization is mainly pointing in one direction $\left(\vartheta=0^{\circ}\right)$, with some small elongated domains pointing in the opposite one (red and light blue colors, respectively). Hence, there is a clear preferential alignment of $\mathbf{M}$ along one axis. After switching the polarization to $P_{\text {up }}$ [Fig. 5(c)] the direction of the magnetization vector changed for a large portion of the probed area (purple color). This happens at both sides of the crack. However, along the fracture opened by the switching, the magnetization appears disordered. We ascribe this local disorder to the effect of dipolar interactions across the crack of the two regions vertically displaced. When the polarization is switched again down [Fig. 5(d), $\left.P_{\text {down }}\right]$ the system goes back to an assembly of domains pointing along the originally preferred axis. The crack, now closed, coincides with a $180^{\circ}$ domain wall.

These data show that by simply reversing the PMN-PT polarization, the average magnetization direction of the $\mathrm{Fe}$ layer is drastically changed in absence of any magnetic fields [32]. It is clear that in the $P_{\text {up }}$ case $\mathbf{M}$ strongly prefers a different orientation compared to the $P_{\text {down }}$ case. From the magnetization vector maps, we calculated the spatially resolved rotation of $\mathbf{M}$. We subdivided the images according to the domain distributions in the $P_{\text {down }}$ state and we plotted the distribution of rotation angles in the histograms of Fig. 5(e) (corresponding to the switch from $P_{\text {down }}$ to $P_{\text {up }}$, subdivided according to the domains in b) and Fig. 5(f) (switch from $P_{\text {up }}$ to $P_{\text {down }}$, subdivided according to the domains in d). By quantifying the rotation of the different magnetic domains, we see that the small domains originally oriented $\vartheta=180^{\circ}$ rotate by $-60^{\circ}$ [Fig. 5(e), blue histogram]. Similarly, the light blue domains observed in Fig. 5(d) originate from a rotation of $65^{\circ}$ [Fig. 5(f), blue histograms]. In the big domain originally oriented $\vartheta=0^{\circ}$ [red color in Fig. 5(b)] M rotates according to a wide distribution of angles. The most represented value is $111^{\circ}$ (although this is not the mean of the distribution, due to its marked asymmetry) as shown by the red histogram in Fig. 5(e). A similar behavior is observed for the red domains 
$\mathrm{Fe} / \mathrm{PMN}_{0.6}-\mathrm{PT}_{0.4}(001)$
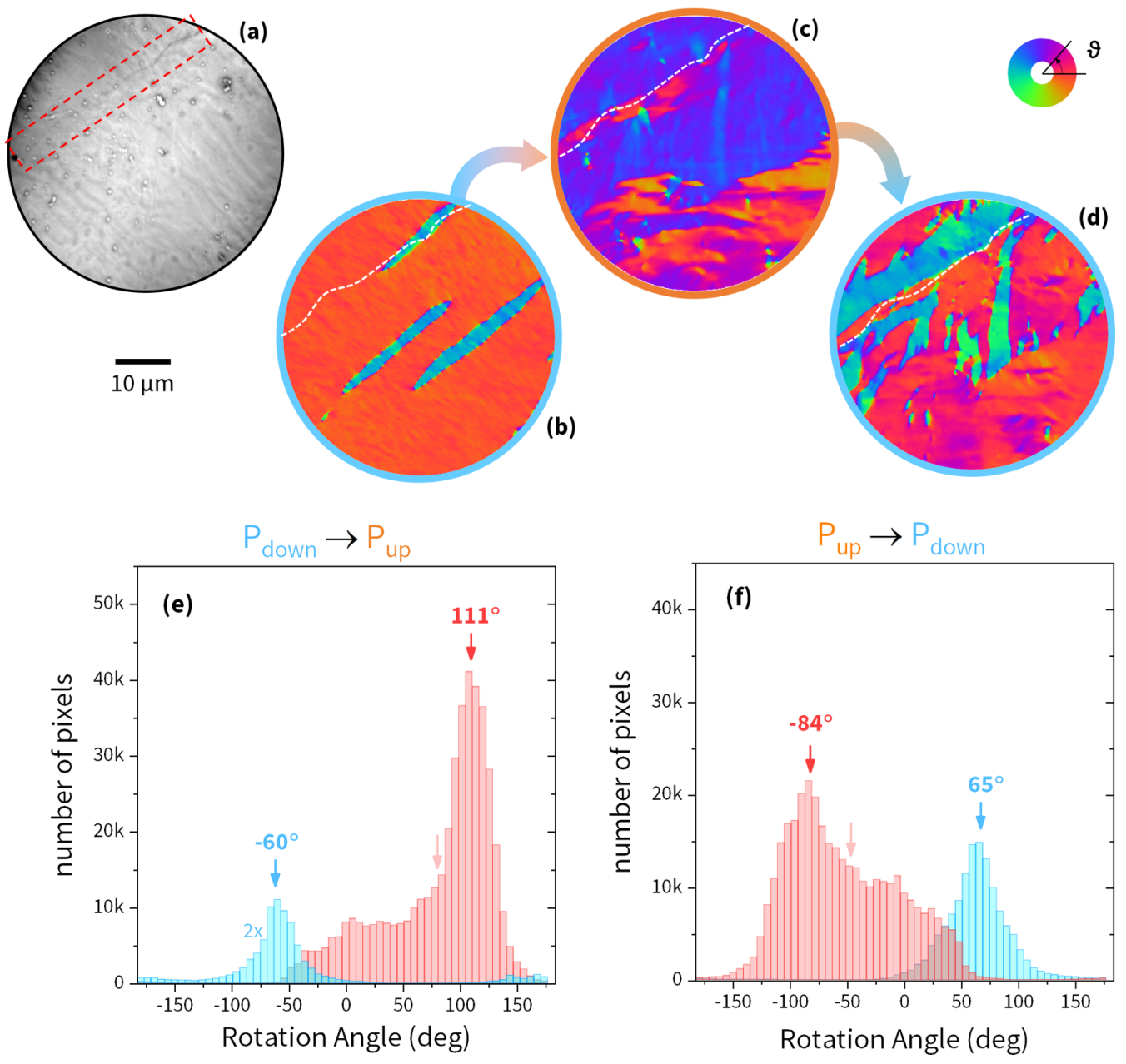

FIG. 5. (a) Polarization-averaged PEEM image acquired at the $\mathrm{Fe} L_{3}$ preedge for an $\mathrm{Fe} / \mathrm{PMN}_{0.6}-\mathrm{PT}_{0.4}(001)$ sample in $P_{\text {up }}$ state. A surface crack is highlighted by the red dashed box. (b) Magnetization vector map for $P_{\text {down }}$ state, with the sample demagnetized. (c) After the first in situ switching to $P_{\text {up }}$ state. (d) After switching back to $P_{\text {down }}$. The position of the crack is marked by a dashed white line. (e) Histogram of the pixel-by-pixel rotation of the magnetization vector, going from (b) to (c). (f) Similar histogram, going from (c) to (d). The histograms were subdivided according to the magnetic domains observed in the $P_{\text {down }}$ state. Bold and light arrows denote the peak and average values of the rotation distributions, respectively.

in Fig. 5(d), which originate from the rotation events shown by the red histogram in Fig. 5(f). Here the peak-value is $-84^{\circ}$, but the distribution is even more asymmetric.

In the second probed region, shown in Fig. 6, the starting magnetic state was fully demagnetized and no surface cracks were observed in the $P_{\text {up }}$ state. These data were collected in parallel with those of Fig. 5, moving from one region to the other before each switch of the polarization. In Fig. 6(a) we show the polarization-averaged intensity map acquired far from the $\mathrm{Fe} L_{3}$ edge, showing a smooth and uniform contrast except from small impurity particles on the surface. In Fig. 6(b), one can see a complex arrangement of magnetic domains in the demagnetized $P_{\text {down }}$ state, with no evident preferential orientation. After switching to the $P_{\text {up }}$ state [Fig. 6(c)], the magnetization points almost uniformly to the same direction $\left(\vartheta=0^{\circ}\right.$, red color). After switching the polarization back to $P_{\text {down }}$, the magnetic configuration returns to a more complex state, partially recovering the initial one, with the presence of some domains oriented along approximately $\vartheta=$ $\pm 90^{\circ}$ [green or blue, Fig. 6(d)]. By repeating the previous quantitative analysis of the rotation of the magnetic domains upon polarization switching, we can see from the histograms in Figs. 6(e) and 6(f) that the rotation distributions are broad (standard deviation on the order of several tens of degrees), and often asymmetric. Therefore, it is difficult to identify a uniform rotation of the magnetic domains by an angle of $\Delta \vartheta$. This is particularly true for the data in Fig. 6(f), i.e., when switching back from $P_{\text {up }}$ to $P_{\text {down }}$. Since in Fig. 6(d) $\mathbf{M}$ is oriented over a wide distribution of angles, the subdivision in domains is somewhat arbitrary. As a result, the histograms in Fig. 6(f) strongly overlap. However, the statistical averages of the rotation distributions (marked by arrows) deviate significantly from the values of $\pm 90^{\circ}$ expected in the purely strain-mediated model. Comparing Figs. 5 and 6, we notice that the preferential orientations induced by $P_{\text {up }}$ are different (about orthogonal) in the two regions investigated. Whatever 
$\mathrm{Fe} / \mathrm{PMN}_{0.6}-\mathrm{PT}_{0.4}(001)$
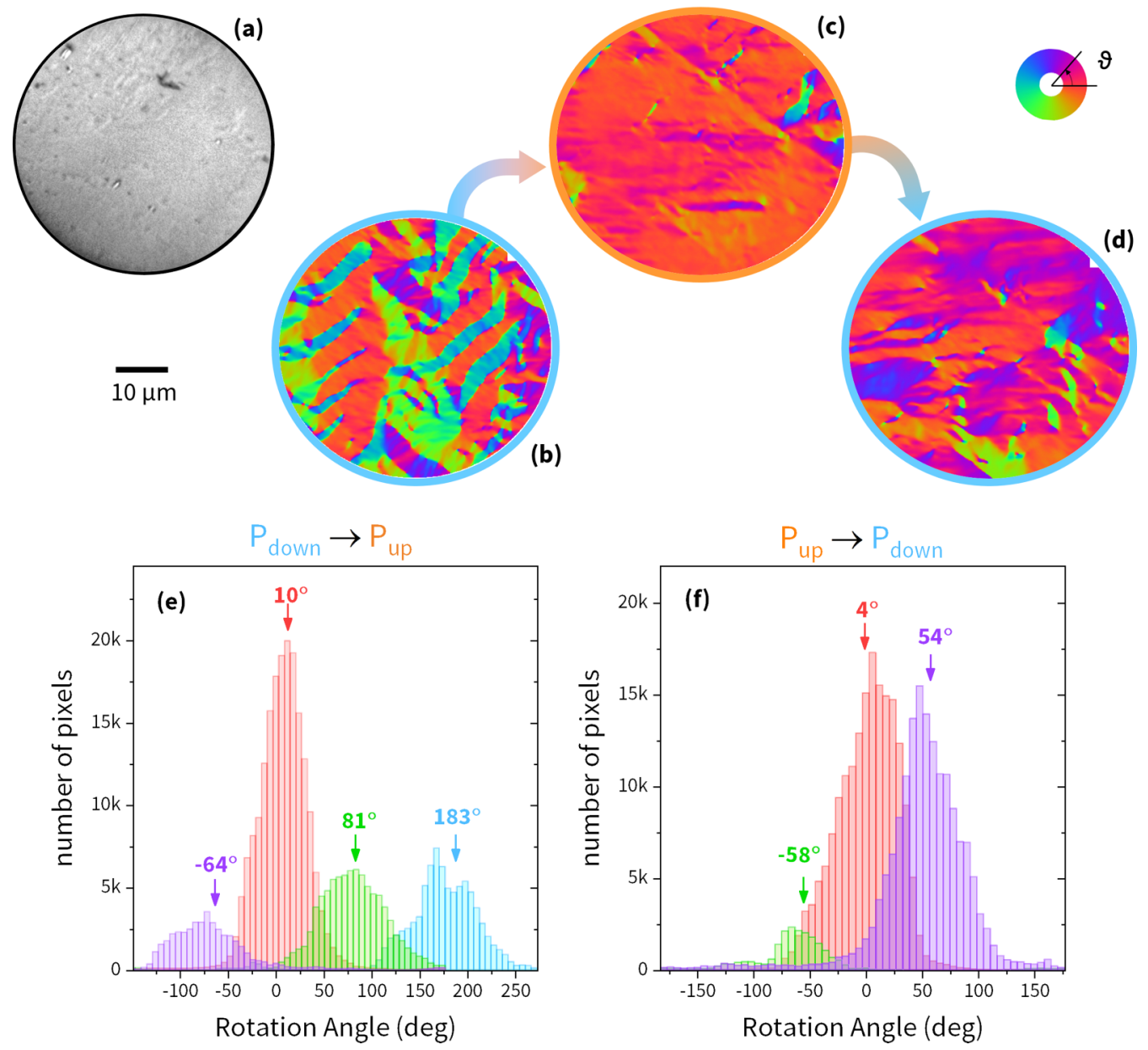

FIG. 6. Data analogous to those of Fig. 5, on a different portion of the same Fe $/ \mathrm{PMN}_{0.6}-\mathrm{PT}_{0.4}(001)$ sample.

the mechanism driving this change, its effect is not uniform all over the surface of the sample, in accordance with the Kerr microscopy data presented above.

Moreover, in the case of Fig. 5 we have observed how the presence of a surface crack locally increases the complexity of the magnetic configuration at remanence. This can be attributed to the breaking of the exchange coupling across the crack, and to a modification of the dipolar interaction between the terraces in the two states. This is a direct proof of how morphologic effects locally play a role in the magnetic configuration of multiferroic heterostructures.

\section{Magnetoelectric coupling in Fe/PMN-PT (011)}

We compared the previous results with similar preliminary experiments on $\mathrm{PMN}_{0.7}-\mathrm{PT}_{0.3}(011)$, which is more commonly used to fabricate multiferroic heterostructures. This substrate displays also an electrically controlled, reversible surface cracking. Therefore, this phenomenon seems not to be limited to a particular cut or composition. However, the pattern of the cracks and their dimensions are quite different for the two cases, in both density and step height. In PMN-PT (011), cracks can be some millimeters long, and are mostly oriented parallel to the [100] direction, separated by fractions of millimeters (see Fig. 7, panel a). Not all the cracks disappear in the $P_{\text {down }}$ state, but only about half of them (panel b). Optical interference profilometry data (panels c, d) show that the step height is a few hundreds of nanometers, which is about one order of magnitude larger than that observed for PMN-PT(001).

We can understand qualitatively the striped pattern of the cracks from crystallographic considerations. For the (011) cut the two in-plane lattice constants are not equivalent: [011] is longer than [100]. We may expect that the internal stresses that build up inside the crystal during the out-of-plane polarization will be anisotropic, resulting in cracking along a preferential direction [41].

Magnetoelectric coupling in Fe/PMN-PT (011) was studied by longitudinal MOKE, measuring hysteresis loops for different orientations of magnetic field $\mathbf{H}$ [Figs. 7(e) and 7(f)]. These measurements probe the magnetic properties averaged on a macroscopic area determined by the size of the laser spot (about $1 \mathrm{~mm}$ in diameter). The effect of switching $\mathbf{P}$ from down to up is to rotate the magnetic anisotropy by $90^{\circ}$, de facto swapping the hard and easy axes. The complete dataset is available in the Supplemental Material file, Fig. S2 [42]. 


$$
\mathrm{PMN}_{0.7}-\mathrm{PT}_{0.3}(011)
$$
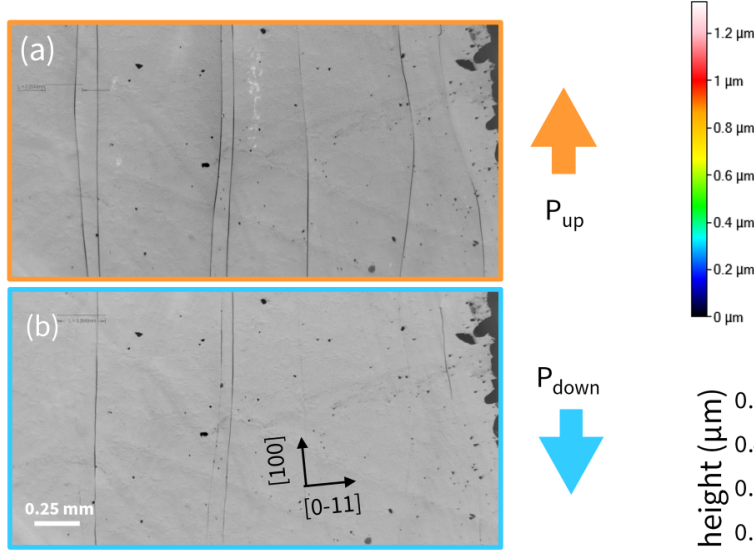
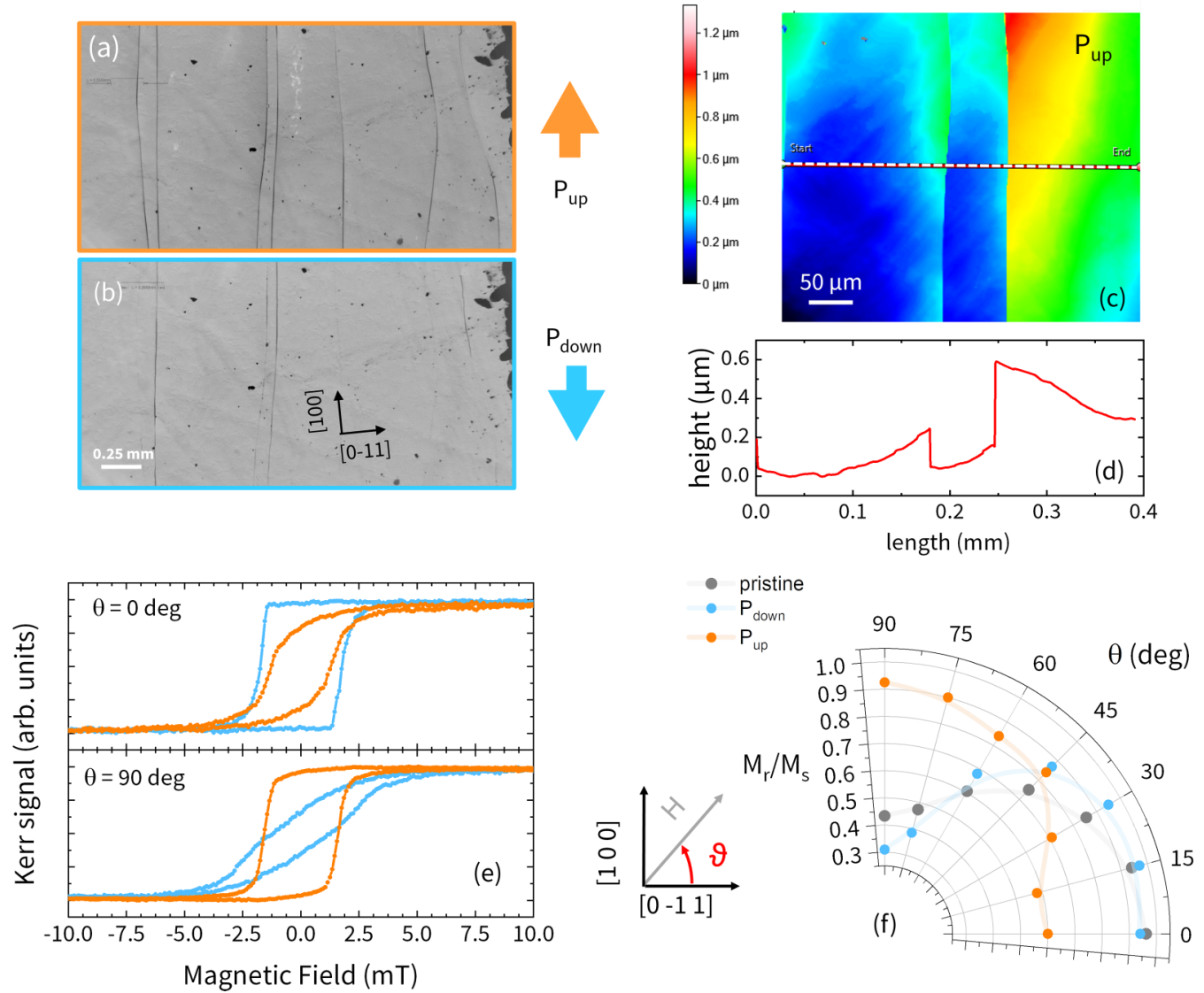

FIG. 7. Changes in morphology and magnetoelectric coupling in PMN-PT(011). (a) Optical microscope image in the cracked state, and

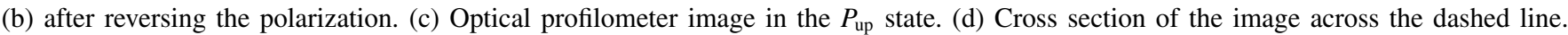
(e) Longitudinal MOKE hysteresis loops for two orthogonal orientations and different polarization state of the substrate. (f) Polar plot of the magnetic remanence $M_{\mathrm{R}}$ normalized to the magnetic saturation $M_{\mathrm{S}}$, for the pristine and polarized cases, as obtained by MOKE.

MOKE measurements were done at zero electric bias after sweeping the voltage up to a value well beyond the coercive field and then back to zero [see Fig. 1(c)]. A similar rotation of the magnetic easy axis in FM/PMN-PT (011) usually requires a precise tuning of the maximum applied voltage close to the ferroelectric coercive field. The phenomenon that we observe is more robust, hence more appealing for applications. XMCD characterizations of the same sample, with a spatial resolution determined by a X-ray spot size of $100 \mu \mathrm{m}$, show that the remanent magnetization changes in the same direction throughout the surface, although varies in intensity (see Supplemental Material file, Fig. S3 [42]).

We have seen that, for both the compositions and substrate cuts studied, cracking of the surface corresponds to a change in magnetic anisotropy. The difference lies in the spatial variation of the effect. In the (011) case, cracking happens in a quite ordered manner, uniformly on a macroscopic scale. Consequently, a net magnetoelectric effects is observed (MOKE data), although there are quantitative differences on the submillimeter scale (probed by XMCD). In the (001) case instead, the cracks are dense, highly irregular, and their shape varies drastically on the $100-\mu \mathrm{m}$ scale. Therefore, magnetoelectric effects vary substantially on the same scale: opposite effects on $\mathbf{M}$ in response to the same electric polarization were observed, according to the mesoscopic area probed and the crack pattern [38]. Another difference lies in the relationship between cracks and easy axis. While in PMN-PT(001) cracking favors an easy axis perpendicular to the cracks, in PMN-PT(011) the opposite was found. Thus, the interplay between morphology and magnetic anisotropy seems to depend strongly on the length scale of the former.

\section{DISCUSSION}

\section{A. Reversible cracking of PMN-PT: Possible origin}

The origin of the observed unusual morphological effect in PMN-PT may reside in the internal stress that develops inside the crystal during electrical poling, probably due to the initial multidomain state. Indeed, fatigue in relaxor ferroelectrics induces preferential cracking along the domain boundaries $[43,44]$. When two adjacent ferroelectric domains switch following different rotation paths, the shear strain may be enough to produce a crack [37]. Therefore, we may expect the cracking pattern to carry an imprint of the pristine distribution of ferroelectric domains. This is however not evident comparing with the reports present in the literature.

The ferroelectric domains in tetragonal $\mathrm{PMN}_{1-x}-\mathrm{PT}_{x}$ (001) $(x>0.4)$ form regions separated by $90^{\circ}$ walls 
alternate in a striped pattern, with a $10-30-\mu \mathrm{m}$ width. This scale matches with the crack separation we observe in some regions. Each stripe is finely subdivided into smaller domains separated by $180^{\circ}$ walls. Moreover, $a-c$ and $a-a$ striped regions are separated on a mesoscopic scale [45]. In rhombohedral $\mathrm{PMN}_{0.7}-\mathrm{PT}_{0.3}(001)$ instead, ferroelectric domains usually form either fingerprintlike patterns with a lateral scale of a fraction of micrometer or irregular stripelike patterns a few micrometers long, identified as formed by $180^{\circ}$ domain walls [46]. Zigzag ("tire-track") patterns were also observed, with a typical lateral scale of $2-3 \mu \mathrm{m}$, and interpreted as copenetrating $71^{\circ} / 109^{\circ}$ domain walls [45]. Such features are not present in the crack pattern observed. For the (011) cut, regions with fingerprintlike or "tweed"-like domain patterns alternate in a striped array [47]. However, the observed crack separation for rhombohedral PMN-PT(011) (i.e., $x=0.3$ ) is way larger than the domain patterns previously reported. These considerations show that it is not straightforward to associate surface cracks with ferroelectric domain walls or vice versa. However, the domain patterns in ferroelectrics are known to depend on the thermal history of the sample, and this may be the key for the observation of reversible cracking.

\section{B. Morphology and magnetic properties}

We have shown that the magnetic properties of Fe/PMNPT heterostructures are strongly influenced by the ferroelectric polarization of the substrate, including its surface morphology. Often magnetoelectric coupling in FM/PMN-PT heterostructures has been described as purely strain mediated. However, some observations clash with this simple hypothesis, due to the complex relation between the ferroelectric domains microstructure and the cracks formation. First, the strong magnetoelectric coupling comes by switching $P$ out of plane, which implies that the $P_{\text {up }}$ and $P_{\text {down }}$ configurations should be structurally- (and strain-) symmetric for both tetragonal PMN-PT(001) and rhombohedral PMN-PT(011). Furthermore, we may intuitively expect different strains in regions separated by cracks, as modeled by Liu et al. [37] They proposed that the origin of the cracking might be the shear strain built up at a ferroelectric domain wall, when one domain is pinned and the other undergoes a $109^{\circ}$ rotation. In this scenario, regions separated by a crack should be subject to a different in-plane strain, and therefore they should display a different magnetic anisotropy, with the easy axes $90^{\circ}$ apart. Our Kerr microscopy analysis shows that this is not the case, since all the stripes react in a similar way upon switching of electrical polarization. PEEM data also show the same preferential orientation of $\mathbf{M}$ in regions separated by a crack (see Fig. 5), and a wide distribution of rotation angles between $P_{\text {up }}$ and $P_{\text {down }}$ (Figs. 5 and 6) different from $\Delta \vartheta= \pm 90^{\circ}$ expected for strain-mediated coupling in rhombohedral PMN-PT(001) $\left( \pm 62^{\circ}\right.$ considering the effect shear strain in rhombohedral PMN-PT (011) [32]). These facts question the intuitive correspondence between cracks, ferroelectric domain walls, and change in magnetic anisotropy. If cracks are formed by some inhomogeneous local force during ferroelectric switching, whatever causes the rotation of magnetic anisotropy has a larger characteristic length scale. Finally, dipolar interactions may play an important role in the observed relation between morphology and magnetic anisotropy. We can try to model two terraces separated by a crack as two interacting pointlike dipoles, separated by a vector $\mathbf{r}$ perpendicular to the crack (see Supplemental Material file [42] for a more detailed discussion). For two interacting magnetic dipoles, the lowest-energy state is when both are parallel and aligned with $\mathbf{r}$. This configuration is lower in energy compared to the one in which the dipoles are parallel/antiparallel but perpendicular to $\mathbf{r}$. Therefore, in this crude approximation, we expect them to orient with $\mathbf{M}$ roughly perpendicular to the crack, in agreement with our observation on $\mathrm{PMN}_{0.6}-\mathrm{PT}_{0.4}(001)$ (see Figs. 3 and 4). Detailed micromagnetic simulations would be necessary to establish if dipolar interactions quantitatively account for the observed rotation of the magnetic easy axis.

In any case, from our set of results it appears that a crucial parameter in Fe/PMN-PT heterostructures is the crack pattern on a micro- to mesoscopic scale. These considerations suggest abandoning the simplified connection between local stress and cracking of PMN-PT.

\section{CONCLUSIONS AND PERSPECTIVES}

Our study addresses the complexity of magnetoelectric coupling in FM/PMN-PT heterostructures, and enlightens the role of morphology. We showed that PMN-PT displays electrically controlled and reversible cracking as a general feature, common to different substrate cuts and compositions. Strong magnetoelectric coupling was observed in all the Fe/PMNPT heterostructures investigated, showing a correlation with their morphological changes. $\mathrm{PMN}_{0.7}-\mathrm{PT}_{0.3}(011)$ can display a relatively ordered cracking pattern on a macroscopic scale, and the effect of the switching of $\mathbf{P}$ on the magnetic properties is homogeneous as well. In $\mathrm{PMN}_{0.6}-\mathrm{PT}_{0.4}(001)$ the cracking pattern is much denser and varies widely on a mesoscopic scale; its switching of $\mathbf{P}$ induces very different changes on the magnetization $\mathbf{M}$, according to the position investigated. Kerr microscopy shows that reversal of $\mathbf{P}$ induces coherent changes on the magnetic hysteresis loop in regions separated by a crack, meaning that the mechanism responsible for magnetoelectric coupling varies on a lateral scale larger than the one responsible for the fracturing, and cracks formations may not be due to $109^{\circ}$ ferroelectric domain rotations. XMCDPEEM studies showed a wide distribution of electrically induced magnetic domain rotation angles, including the effect of dipolar fields in the proximity of a crack. In general, the observed phenomenon is a voltage-controlled modification of the magnetic anisotropy energy. However, the coexistence of reversible morphological changes and lattice strain, and their correlation to the magnetic anisotropy, makes the magnetoelectric coupling picture more intricate than what is generally reported. Deepening the understanding of reversible cracking of PMT-PT is necessary to tailor the properties of multiferroic heterostructures for realistic applications, and magnetoelectric devices based on purely morphological effects can be envisioned [48].

\section{ACKNOWLEDGMENTS}

This work has been performed in the framework of the Nanoscience Foundry and Fine Analysis (NFFA-MIUR 
Italy Progetti Internazionali) project. This work was partially performed at Polifab, the micro and nanofabrication facility of Politecnico di Milano. We acknowledge Diamond Light Source for the provision of beamtime under Proposal No. SI18810. C.R. acknowledges the support by Fondazione Cariplo and Regione Lombardia, Grant No. 20171622 (ECOS).

\section{APPENDIX: EXPERIMENTAL METHODS}

All the experiments here reported were performed at room temperature.

\section{Sample preparation}

Substrates of $\mathrm{PMN}_{0.6}-\mathrm{PT}_{0.4}(001)$ (purchased from SurfaceNet $\mathrm{GmbH}$ ) and $\mathrm{PMN}_{0.7}-\mathrm{PT}_{0.3}(011)$ (from Testbourne LTD) were cleaned and rinsed in ultrasonic bath with acetone, ethanol, and deionized water and finally introduced in the deposition chamber [49]. Here an Fe film of $4 \mathrm{~nm}$ was grown by electron-beam evaporation on the substrates, kept at room temperature. The samples were then capped in situ by 3-5 nm of either MgO (for XMCD-PEEM, evaporated from a single crystal by electron bombardment) or $\mathrm{Au}$ (for Kerr microscopy, deposited by electron-beam evaporation from a dedicated crucible). In the sample for Kerr microscopy characterization, an interlayer of $\mathrm{SiO}_{2}(10 \mathrm{~nm})$ was deposited between the substrate and the Fe film to remove any eventual substrate-induced texture in the ferromagnetic layer. Details on the deposition conditions can be found in Ref. [38].

\section{Morphological characterization}

The topographic characterization of the (001)-oriented samples was done by Atomic Force Microscopy (AFM) measurements in tapping mode, acquired with an A100 microscope of A.P.E. Research. The samples were measured at ambient pressure and temperature, using the sample holder connections in the measurement position to switch the electric polarization of the substrates. The AFM tip was lifted during the application of the bias to avoid surface damaging during the morphological transitions. All measurements were done under no voltage applied, after switching the polarization, using cantilevers with stiffness of $40 \mathrm{Nm}$ and length of $125 \mu \mathrm{m}$. On (011)-oriented substrates, the morphology was characterized with a three-dimensional (3D) noncontact optical profilometer (Filmetrics) using a 50- $\mu \mathrm{m}$ field of view in white-light interferometry mode. Finally, optical microscopy was used to visualize large surface areas in the two polarization states. Here we employed an HQ Graphene microscope for PMN-PT (011) substrates and the Kerr microscope for PMN-PT (001) substrates.

\section{PEEM measurements}

Photoemission microscopy (PEEM) experiments were performed at the I06 beamline of Diamond Light Source. Before being introduced in the experimental chamber, the sample was demagnetized by applying an alternating decreasing magnetic field. In the PEEM experiment, the x-ray beam hits from an angle of $16^{\circ}$ with the surface of the sample. Secondary electron emission from the sample was imaged with an Elmitec SPELEEM-III microscope, with a 50- $\mu \mathrm{m}$ field of view. Measuring an XMCD asymmetry image for two orthogonal sample orientations allows reconstructing a 2D vector map of the magnetization. A proper dedicated sample holder allows for application of a voltage up to $300 \mathrm{~V}$ across the sample thickness in situ [50]. Magnetization vector maps were measured after switching the polarization up or down, with zero applied electric field. No magnetic field was applied during the PEEM experiment.

\section{MOKE and Kerr microscopy}

Magnetic hysteresis loops were collected by longitudinal magneto-optical Kerr effect. We employed a blue laser (wavelength $435 \mathrm{~nm}$ ) with $s$ polarization, and the reflected intensity was modulated by a photoelastic modulator (PEM) at $50 \mathrm{kHz}$ before passing through an analyzer with the axis approximately at $45^{\circ}$ from the plane of incidence. The signal coming from the detector was fed to a lock-in amplifier, which uses the PEM signal as a reference [49].

Kerr microscopy measurements were performed at the Nanomagnetism Laboratory of the PoliFab laboratory of the Department of Physics at Politecnico di Milano (Polifab), using an optical wide-field polarization microscope (Zeiss Axiotron II) customized in-house for Kerr microscopy. All the measurements were performed in longitudinal configuration. Images are acquired by a high-resolution, high-sensitivity, and low-noise digital complementary metal-oxide semiconductor camera by Hamamatsu (ORCA-spark C33662-58U). The magnetic hysteresis loops are obtained by selecting an arbitrary region of interest and thus integrating the intensity collected by the corresponding pixels using the camera as a conventional photodetector. The signal for each pixel is proportional to the magnetization along the sensitivity direction, which is parallel to the externally applied magnetic field in these measurements. The electrical polarization of the substrate can be switched without removing the sample from the microscope, thanks to a specific mounting.
[1] M. Bibes and A. Barthélémy, Multiferroics: Towards a magnetoelectric memory, Nat. Mater. 7, 425 (2008).

[2] J. Akerman, Toward a universal memory, Science 308, 508 (2005).

[3] S. Manipatruni, D. E. Nikonov, C. C. Lin, T. A. Gosavi, H. Liu, B. Prasad, Y. L. Huang, E. Bonturim,
R. Ramesh, and I. A. Young, Scalable energy-efficient magnetoelectric spin-orbit logic, Nature (London) 565, 35 (2019).

[4] C. M. Leung, J. Li, D. Viehland, and X. Zhuang, A review on applications of magnetoelectric composites: From heterostructural uncooled magnetic sensors, energy harvesters to highly 
efficient power converters, J. Phys. D: Appl. Phys. 51, 263002 (2018).

[5] J. F. Scott, Multiferroic memories, Nat. Mater. 6, 256 (2007).

[6] M. Fiebig, T. Lottermoser, D. Meier, and M. Trassin, The evolution of multiferroics, Nat. Rev. Mater. 1, 16046 (2016).

[7] N. A. Hill, Why are there so few magnetic ferroelectrics? J. Phys. Chem. B 104, 6694 (2000).

[8] J. F. Scott, Room-Temperature Multiferroic Magnetoelectrics, NPG Asia Materials 5, e72 (2013).

[9] T. H. E. Lahtinen and S. Van Dijken, Temperature control of local magnetic anisotropy in multiferroic $\mathrm{CoFe} / \mathrm{BaTiO}_{3}$, Appl. Phys. Lett. 102, 112406 (2013).

[10] A. Brandlmaier, S. Geprägs, G. Woltersdorf, R. Gross, and S. T. B. Goennenwein, Nonvolatile, reversible electric-field controlled switching of remanent magnetization in multifunctional ferromagnetic/ferroelectric hybrids, J. Appl. Phys. 110, 043913 (2011).

[11] M. Ghidini, F. Maccherozzi, X. Moya, L. C. Phillips, W. Yan, J. Soussi, N. Métallier, M. E. Vickers, N.-J. Steinke, R. Mansell, C. H. W. Barnes, S. S. Dhesi, and N. D. Mathur, Perpendicular local magnetization under voltage control in Ni films on ferroelectric $\mathrm{BaTiO}_{3}$ substrates, Adv. Mater. 27, 1460 (2015).

[12] R. O. Cherifi, V. Ivanovskaya, L. C. Phillips, A. Zobelli, I. C. Infante, E. Jacquet, V. Garcia, S. Fusil, P. R. Briddon, N. Guiblin, A. Mougin, A. A. Ünal, F. Kronast, S. Valencia, B. Dkhil, A. Barthélémy, and M. Bibes, Electric-field control of magnetic order above room temperature, Nat. Mater. 13, 345 (2014).

[13] S. Brivio, D. Petti, R. Bertacco, and J. C. Cezar, Electric field control of magnetic anisotropies and magnetic coercivity in $\mathrm{Fe} / \mathrm{BaTiO}_{3}(001)$ heterostructures, Appl. Phys. Lett. 98, 092505 (2011).

[14] L. Baldrati, C. Rinaldi, A. Manuzzi, M. Asa, L. Aballe, M. Foerster, N. Biškup, M. Varela, M. Cantoni, and R. Bertacco, Electrical switching of magnetization in the artificial multiferroic $\mathrm{CoFeB} / \mathrm{BaTiO}_{3}$, Adv. Electron. Mater. 2, 1600085 (2016).

[15] G. Radaelli, D. Petti, E. Plekhanov, I. Fina, P. Torelli, B. R. Salles, M. Cantoni, C. Rinaldi, D. Gutiérrez, G. Panaccione, M. Varela, S. Picozzi, J. Fontcuberta, and R. Bertacco, Electric control of magnetism at the $\mathrm{Fe} / \mathrm{BaTiO}_{3}$ interface, Nat. Commun. 5, 3404 (2014).

[16] N. A. Spaldin and R. Ramesh, Advances in magnetoelectric multiferroics, Nat. Mater. 18, 203 (2019).

[17] C. Song, B. Cui, F. Li, X. Zhou, and F. Pan, Recent progress in voltage control of magnetism: Materials, mechanisms, and performance, Prog. Mater. Sci. 87, 33 (2017).

[18] J. M. Hu, L. Q. Chen, and C. W. Nan, Multiferroic heterostructures integrating ferroelectric and magnetic materials, Adv. Mater. 28, 15 (2016).

[19] F. Matsukura, Y. Tokura, and H. Ohno, Control of magnetism by electric fields, Nat. Nanotechnol. 10, 209 (2015).

[20] S. Fusil, V. Garcia, A. Barthélémy, and M. Bibes, Magnetoelectric devices for spintronics, Annu. Rev. Mater. Res. 44, 91 (2014).

[21] A. A. Bokov and Z. G. Ye, Recent progress in relaxor ferroelectrics with perovskite structure, J. Mater. Sci. 41, 31 (2006).

[22] B. Noheda, D. E. Cox, G. Shirane, J. Gao, and Z. G. Ye, Phase diagram of the ferroelectric relaxor (1-x) $\mathrm{PbMg}_{1 / 3} \mathrm{Nb}_{2 / 3} \mathrm{O}_{3}-x \mathrm{PbTiO}_{3}$, Phys. Rev. B 66, 054104 (2002).

[23] Y. Zhang, D. Xue, H. Wu, X. Ding, T. Lookman, and X. Ren, Adaptive ferroelectric state at morphotropic phase boundary: Coexisting tetragonal and rhombohedral phases, Acta Mater. 71, 176 (2014).

[24] J. Heidler, C. Piamonteze, R. V Chopdekar, M. A. UribeLaverde, A. Alberca, M. Buzzi, A. Uldry, B. Delley, C. Bernhard, and F. Nolting, Manipulating magnetism in $\mathrm{La}_{0.7} \mathrm{Sr}_{0.3} \mathrm{MnO}_{3}$ via piezostrain, Phys. Rev. B 91, 024406 (2015).

[25] H. Yan, Z. Feng, S. Shang, X. Wang, Z. Hu, J. Wang, Z. Zhu, H. Wang, Z. Chen, H. Hua, W. Lu, J. Wang, P. Qin, H. Guo, X. Zhou, Z. Leng, Z. Liu, C. Jiang, M. Coey, and Z. Liu, A piezoelectric, strain-controlled antiferromagnetic memory insensitive to magnetic fields, Nat. Nanotechnol. 14, 131 (2019).

[26] H. B. Vasili, D. Pesquera, M. Valvidares, P. Gargiani, E. Pellegrin, F. Bondino, E. Magnano, A. Barla, and J. Fontcuberta, In operando adjustable orbital polarization in $\mathrm{LaNiO}_{3}$ thin films, Phys. Rev. Mater. 4, 044404 (2020).

[27] B. Zhi, G. Gao, H. Xu, F. Chen, X. Tan, P. Chen, L. Wang, and W. Wu, Electric-field-modulated nonvolatile resistance switching in $\mathrm{VO}_{2} / \mathrm{PMN}-\mathrm{PT}(111)$ heterostructures, ACS Appl. Mater. Interfaces 6, 4603 (2014).

[28] P. Zhao, M. Bao, A. Bur, J. L. Hockel, K. Wong, K. P. Mohanchandra, C. S. Lynch, and G. P. Carman, Domain engineered switchable strain states in ferroelectric (011) $\left[\mathrm{Pb}\left(\mathrm{Mg}_{1 / 3} \mathrm{Nb}_{2 / 3}\right) \mathrm{O}_{3}\right]_{(1-x)}-\left[\mathrm{PbTiO}_{3}\right]_{x}(\mathrm{PMN}-\mathrm{PT}, x \approx 0.32)$ Single Crystals, J. Appl. Phys. 109, 124101 (2011).

[29] J. Heidler, M. Fechner, R. V Chopdekar, C. Piamonteze, J. Dreiser, C. A. Jenkins, E. Arenholz, S. Rusponi, H. Brune, N. A. Spaldin, and F. Nolting, Magnetoelectroelastic control of magnetism in an artificial multiferroic, Phys. Rev. B 94, 014401 (2016).

[30] M. Buzzi, R. V Chopdekar, J. L. Hockel, A. Bur, T. Wu, N. Pilet, P. Warnicke, G. P. Carman, L. J. Heyderman, and F. Nolting, Single Domain Spin Manipulation by Electric Fields in Strain Coupled Artificial Multiferroic Nanostructures, Phys. Rev. Lett. 111, 027204 (2013).

[31] M. Ghidini, B. Zhu, R. Mansell, R. Pellicelli, A. Lesaine, X. Moya, S. Crossley, B. Nair, F. Maccherozzi, C. H. W. Barnes, R. P. Cowburn, S. S. Dhesi, and N. D. Mathur, Voltage control of magnetic single domains in Ni discs on ferroelectric $\mathrm{BaTiO}_{3}$, J. Phys. D: Appl. Phys. 51, 224007 (2018).

[32] M. Ghidini, R. Mansell, F. Maccherozzi, X. Moya, L. C. Phillips, W. Yan, D. Pesquera, C. H. W. Barnes, R. P. Cowburn, J. Hu, S. S. Dhesi, and N. D. Mathur, Shear-strain-mediated magnetoelectric effects revealed by imaging, Nat. Mater. 18, 840 (2019).

[33] S. Zhang, Y. G. Zhao, P. S. Li, J. J. Yang, S. Rizwan, J. X. Zhang, J. Seidel, T. L. Qu, Y. J. Yang, Z. L. Luo, Q. He, T. Zou, Q. P. Chen, J. W. Wang, L. F. Yang, Y. Sun, Y. Z. Wu, X. Xiao, X. F. Jin, J. Huang, C. Gao, X. F. Han, and R. Ramesh, Electric-Field Control of Nonvolatile Magnetization in $\mathrm{Co}_{40} \mathrm{Fe}_{40} \mathrm{~B}_{20} / \mathrm{Pb}\left(\mathrm{Mg}_{1 / 3} \mathrm{Nb}_{2 / 3}\right)_{0.7} \mathrm{Ti}_{0.3} \mathrm{O}_{3}$ Structure at Room Temperature, Phys. Rev. Lett. 108, 137203 (2012).

[34] P. Li, Y. Zhao, S. Zhang, A. Chen, D. Li, J. Ma, Y Liu, D. T. Pierce, J. Unguris, H. G. Piao, H. Zhang, M. Zhu, X. Zhang, X. Han, M. Pan, and C. W. Nan, Spatially resolved ferroelectric domain-switching-controlled 
magnetism in $\mathrm{Co}_{40} \mathrm{Fe}_{40} \mathrm{~B}_{20} / \mathrm{Pb}\left(\mathrm{Mg}_{1 / 3} \mathrm{Nb}_{2 / 3}\right)_{0.7} \mathrm{Ti}_{0.3} \mathrm{O}_{3}$ multiferroic heterostructure, ACS Appl. Mater. Interfaces 9, 2642 (2017).

[35] Y. Ba, Y. Liu, P. Li, L. Wu, J. Unguris, D. T. Pierce, D. Yang, C. Feng, Y. Zhang, H. Wu, D. Li, Y. Chang, J. Zhang, X. Han, J. Cai, C.-W. Nan, and Y. Zhao, Spatially resolved electric-field manipulation of magnetism for $\mathrm{CoFeB}$ mesoscopic discs on ferroelectrics, Adv. Funct. Mater. 28, 1706448 (2018).

[36] S. Zhang, Q. Chen, Y. Liu, A. Chen, L. Yang, P. Li, Z. S. Ming, Y. Yu, W. Sun, X. Zhang, Y. Zhao, Y. Sun, and Y. Zhao, Strain-mediated coexistence of volatile and nonvolatile converse magnetoelectric effects in $\mathrm{Fe} / \mathrm{Pb}\left(\mathrm{Mg}_{1 / 3} \mathrm{Nb}_{2 / 3}\right)_{0.7} \mathrm{Ti}_{0.3} \mathrm{O}_{3}$ heterostructure, ACS Appl. Mater. Interfaces 9, 20637 (2017).

[37] Z. Q. Liu, J. H. Liu, M. D. Biegalski, J. M. Hu, S. L. Shang, Y. Ji, J. M. Wang, S. L. Hsu, A. T. Wong, M. J. Cordill, B. Gludovatz, C. Marker, H. Yan, Z. X. Feng, L. You, M. W. Lin, T. Z. Ward, Z. K. Liu, C. B. Jiang, L. Q. Chen, R. O. Ritchie, H. M. Christen, and R. Ramesh, Electrically reversible cracks in an intermetallic film controlled by an electric field, Nat. Commun. 9, 41 (2018).

[38] G. Vinai, F. Motti, V. Bonanni, A. Y. Petrov, S. Benedetti, C. Rinaldi, M. Stella, D. Cassese, S. Prato, M. Cantoni, G. Rossi, G. Panaccione, and P. Torelli, Reversible modification of ferromagnetism through electrically controlled morphology, Adv. Electron. Mater. 5, 1900150 (2019).

[39] R. C. Temple, T. P. Almeida, J. R. Massey, K. Fallon, R. Lamb, S. A. Morley, F. Maccherozzi, S. S. Dhesi, D. McGrouther, S. McVitie, T. A. Moore, and C. H. Marrows, Antiferromagneticferromagnetic phase domain development in nanopatterned FeRh islands, Phys. Rev. Mater. 2, 104406 (2018).

[40] W. Saenrang, B. A. Davidson, F. Maccherozzi, J. P. Podkaminer, J. Irwin, R. D. Johnson, J. W. Freeland, J. Íñiguez, J. L. Schad, K. Reierson, J. C. Frederick, C. A. F. Vaz, L. Howald, T. H. Kim, S. Ryu, M. V. Veenendaal, P. G. Radaelli, S. S. Dhesi, M. S. Rzchowski, and C. B. Eom, Deterministic and robust room-temperature exchange coupling in monodomain multiferroic $\mathrm{BiFeO}_{3}$ heterostructures, Nat. Commun. 8, 1583 (2017).

[41] F. Fang, W. Yang, F. C. Zhang, and H. Qing, Electric fieldinduced crack growth and domain-structure evolution for [100]and [101]-oriented $72 \% \mathrm{~Pb}\left(\mathrm{Mg}_{1 / 3} \mathrm{Nb}_{2 / 3}\right) \mathrm{O}_{3}-28 \% \mathrm{PbTiO}_{3}$ ferroelectric single crystals, J. Mater. Res. 23, 3387 (2008).
[42] See Supplemental Material at http://link.aps.org/supplemental/ 10.1103/PhysRevMaterials.4.114418 for spatially resolved magnetization rotation maps obtained by XMCD-PEEM in $\mathrm{Fe} / \mathrm{PMN}-\mathrm{PT}(001)$, longitudinal MOKE hysteresis loops of Fe/PMN-PT(011), XMCD characterization of Fe/PMNPT(011), details on PEEM data analysis, and considerations on dipolar interactions.

[43] X. Tan, Z. Xu, J. K. Shang, and P. Han, Direct observations of electric field-induced domain boundary cracking in $\langle 001\rangle$, oriented piezoelectric $\mathrm{Pb}\left(\mathrm{Mg}_{1 / 3} \mathrm{Nb}_{2 / 3}\right) \mathrm{O}_{3}-\mathrm{PbTiO}_{3}$ single crystal, Appl. Phys. Lett. 77, 1529 (2000).

[44] S. Zhang, J. Luo, F. Li, R. J. Meyer Jr., W. Hackenberger, and T. R. Shrout, Polarization fatigue in $\mathrm{Pb}\left(\mathrm{In}_{0.5} \mathrm{Nb}_{0.5}\right) \mathrm{O}_{3}-\mathrm{Pb}\left(\mathrm{Mg}_{1 / 3} \mathrm{Nb}_{2 / 3}\right) \mathrm{O}_{3}-\mathrm{PbTiO}_{3}$ Single Crystals, Acta Mater. 58, 3773 (2010).

[45] M. C. Shin, S. J. Chung, S. G. Lee, and R. S. Feigelson, Growth and observation of domain structure of lead magnesium Niobate-Lead Titanate single crystals, J. Cryst. Growth 263 , 412 (2004).

[46] H. R. Zeng, H. F. Yu, R. Q. Chu, G. R. Li, H. S. Luo, and Q. R. Yin, Domain orientation imaging of PMN-PT single crystals by vertical and lateral piezoresponse force microscopy, J. Cryst. Growth 267, 194 (2004).

[47] H. F. Yu, H. R. Zeng, R. Q. Chu, G. R. Li, H. S. Luo, Z. K. $\mathrm{Xu}$, and Q. R. Yin, Distribution and formation mechanism of the domain structure in PMN-33\% PT single crystals, J. Phys. D: Appl. Phys. 37, 2914 (2004).

[48] V. Polewczyk, G. Vinai, F. Motti, S. Dal Zilio, P. Capaldo, M. Sygletou, S. Benedetti, G. Rossi, and P. Torelli, Original design of a patterned multiferroic heterostructure for electrical control of the magnetic shape anisotropy, J. Magn. Magn. Mater. 507, 166816 (2020)

[49] G. Vinai, F. Motti, A. Petrov, V. Polewczyk, V. Bonanni, R. Edla, R. Ciprian, B. Gobaut, J. Fuji, A. Deluisa, D. Benedetti, F. Salvador, A. Fondacaro, G. Rossi, G. Panaccione, B. Davidson, and P. Torelli, MBE cluster: An integrated UHV apparatus for MBE growth and in-situ characterization of complex materials, Rev. Sci. Instrum. 91, 085109 (2020).

[50] F. Motti, G. Vinai, A. Petrov, B. A. Davidson, B. Gobaut, A. Filippetti, G. Rossi, G. Panaccione, and P. Torelli, Straininduced magnetization control in an oxide multiferroic heterostructure, Phys. Rev. B 97, 094423 (2018). 